\title{
The Influence of Climate and Livestock Reservoirs on Human Cases of Giardiasis
}

\author{
Ariel Brunn, ${ }^{1}$ David N. Fisman, ${ }^{2}$ Jan M. Sargeant, ${ }^{1,3,4}$ and Amy L. Greer ${ }^{1,3}$ \\ ${ }^{1}$ Department of Population Medicine, Ontario Veterinary College, University of Guelph, 50 Stone Road East, Guelph, ON N1G 2W1, Canada \\ ${ }^{2}$ Department of Epidemiology, Dalla Lana School of Public Health, University of Toronto, Toronto, ON, Canada \\ ${ }^{3}$ Centre for Public Health and Zoonoses, University of Guelph, Guelph, ON, Canada \\ ${ }^{4}$ Arrell Food Institute, University of Guelph, Guelph, ON, Canada
}

\begin{abstract}
Giardia duodenalis is an intestinal parasite which causes diarrhoeal illness in people. Zoonotic subtypes found in livestock may contribute to human disease occurrence through runoff of manure into multiuse surface water. This study investigated temporal associations among selected environmental variables and $G$. duodenalis occurrence in livestock reservoirs on human giardiasis incidence using data collected in the Waterloo Health Region, Ontario, Canada. The study objectives were to: (1) evaluate associations between human cases and environmental variables between 1 June 2006 and 31 December 2013, and (2) evaluate associations between human cases, environmental variables and livestock reservoirs using a subset of this time series, with both analyses controlling for seasonal and long-term trends. Human disease incidence exhibited a seasonal trend but no annual trend. A Poisson multivariable regression model identified an inverse association with water level lagged by 1 month (IRR $=0.10,95 \%$ CI $0.01,0.85, P<0.05$ ). Case crossover analysis found varying associations between lagged variables including livestock reservoirs (1 week), mean air temperature (3 weeks), river water level ( 1 week) and flow rate (1 week), and precipitation (4 weeks). This study contributes to our understanding of epidemiologic relationships influencing human giardiasis cases in Ontario, Canada.
\end{abstract}

Keywords: Giardia, Giardiasis, Climate, Watershed, One health, Case crossover, Poisson

\section{INTRODUCTION}

Enteric illness caused by the intestinal parasite Giardia duodenalis $(G d)$ is reportable in Canada. The parasite is the third-leading cause of enteric disease in the province of

Electronic supplementary material: The online version of this article (https://doi. org/10.1007/s10393-018-1385-7) contains supplementary material, which is available to authorized users.

Published online: October 22, 2018

Correspondence to: Amy L. Greer, e-mail: agreer@uoguelph.ca
Ontario with an average provincial incidence of 9.9 cases per 100000 person-years reported between 2011 and 2015 (Public Health Ontario 2017). Many patients remain asymptomatic or are only transiently afflicted causing underreporting of the true prevalence. Symptomatic patients contribute to the estimated 89.5 million disabilityadjusted-life-years lost world-wide due to diarrheal disease (Conlan and Lal 2015; Murray et al. 2012). However, correlations between socioeconomic factors such as lack of plumbing and an increased risk of infection may explain 
why giardiasis has a higher prevalence in lower-income countries (Silva et al. 2009). Symptomatic cases of giardiasis may exhibit mild abdominal discomfort, diarrhoea, vomiting and weight loss, however, most infections are selfresolving.

$G d$ is composed of eight assemblages $(\mathrm{A}-\mathrm{H})$, which are genetic groupings with varying degrees of host-specific pathogenicity. Assemblages A and B primarily cause disease in humans (Yaoyu and Xiao 2011) but have zoonotic potential as they have been isolated from cattle, swine, dogs and cats, as well as some wild mammals (Cacciò and Ryan 2008; Farzan et al. 2011; Yaoyu and Xiao 2011). Assemblage types $\mathrm{C}-\mathrm{H}$ have limited host ranges of domestic and wild animals, but rare occurrences in people have been reported (Yaoyu and Xiao 2011). Zoonotic assemblages in livestock contribute to human disease through direct exposure or through manure contaminated water sources used for recreation or drinking water (Hunter and Thompson 2005; Farzan et al. 2011; Abdel-Moein and Saeed 2016). Ontario dairy cattle and pigs have been shown to harbour zoonotic subtypes of $G d$ (Coklin et al. 2007; Dixon et al. 2011; Farzan et al. 2011) and cysts shed in host faeces are immediately infective in the environment. The low specific gravity of cysts along with high precipitation enhances transport from soil into water sources (Erickson and Ortega 2006; Wilkes et al. 2011). Cysts are well adapted for wet and humid conditions, remaining infective in water with a temperature of $0-4{ }^{\circ} \mathrm{C}$ for 84 days (Yaoyu and Xiao 2011). Warmer air temperatures lead to increased recreational use of water sources by people and hydrological characteristics of watersheds such as water flow and level can affect the suspension and sedimentation rate of cysts in surface waters (Erickson and Ortega 2006) facilitating Giardia cyst ingestion by people (Thomas et al. 2006; Peng et al. 2008). The most common risk factors for human infection in Ontario include ingestion of contaminated water (e.g. drinking or recreational water), direct transmission via the faecal-oral route from infected people or animals, or consumption of contaminated food such as unwashed vegetables (Public Health Ontario 2017). Although outbreaks of giardiasis have been reported globally, they are uncommon in Ontario with a recent descriptive study suggesting that $99 \%$ of Giardia cases are not linked epidemiologically (Keegan et al. 2009).

The seasonal pattern of human Giardia incidence has been reported with annual peaks in the late summer and early autumn (Addiss et al. 1992; Greig et al. 2001; Naumova et al. 2007; Keegan et al. 2009; Lal et al. 2012). This seasonal fluctuation, combined with the ability of Giardia to persist in the environment, is highly suggestive of a transmission cycle that is sensitive to climate and hydrological factors (Fisman 2007; Greer et al. 2008; Ng et al. 2008). Therefore, our a priori hypothesis is that environmental and hydrological conditions are associated with the occurrence of sporadic human Giardia cases within southern Ontario. Understanding associations between environment, livestock reservoirs, and human disease may enhance prevention and control strategies. In this respect, the use of a one health approach is appropriate for the study of Giardia to avoid simplifying complex relationships (Bouley et al. 2014). The one health concept is a framework that recognizes the interconnectedness between the health of humans, animals and the environment (Bouley et al. 2014). The objectives of this study were to identify temporal associations between environmental conditions, livestock Giardia reservoirs and human giardiasis incidence using an integrated time series (2006-2013) from the Waterloo Health Region (WHR) in Ontario, Canada, and to compare the results obtained using two different methods that account for confounding due to seasonality and annual trends.

\section{MetHOdS}

\section{Sources of Data}

\section{Human Case Data}

The Canadian province of Ontario is made up of 36 public health units and all confirmed cases of $G d$ are reported to the provincial Integrated Public Health Information System (iPHIS). The WHR encompasses part of the multi-use Grand River watershed located in southwestern Ontario (Fig. 1). In 2011, the $1369 \mathrm{~km}^{2}$ region was densely populated with 507096 people, 815 cattle farms and 210 swine farms (Statistics Canada 2011).

Confirmed cases of $G d$ are reported to iPHIS based on the finding of cysts or trophozoites in stool samples from patients with compatible clinical signs (after 2009, demonstration of $G d$ antigen by enzyme immunoassay or immunochromatographic test was also accepted in place of microscopy) (Ontario Ministry of Health and Long-Term Care 2016). The case report date could be the date of symptom onset, the specimen submission date, the lab report date or the date the case was reported to the data- 


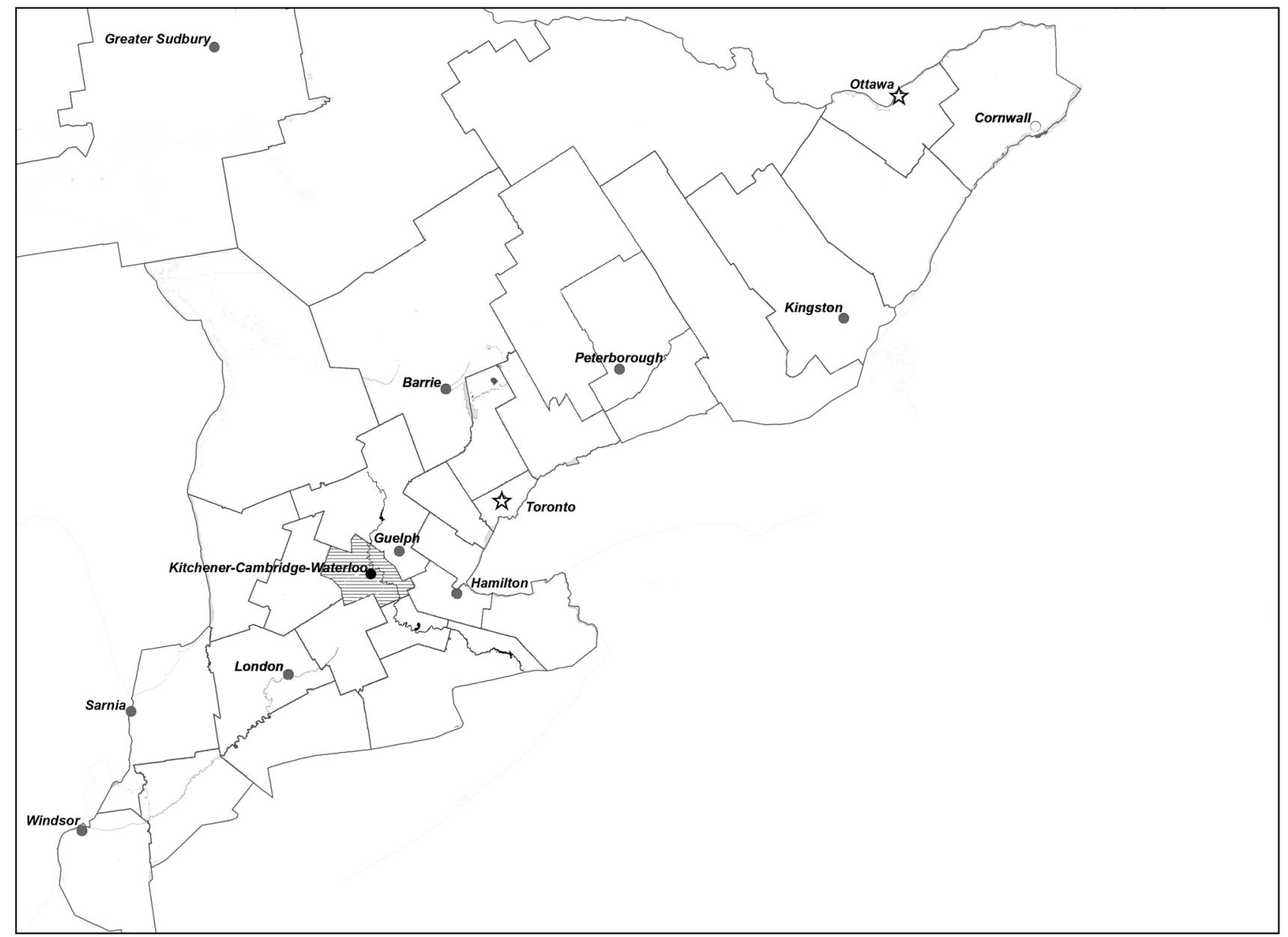

Figure 1. Map of the Waterloo Health Region, Ontario, Canada (WHR—shaded region) where the observational data were collected. The Grand River can be seen as a river that flows from north to south through the health region. * = Location of Waterloo International Airport where Environment Canada weather conditions (mean temperature, total precipitation) were collected; $\neq=$ location of Doon, Waterloo County, where Environment and Climate Change Canada hydrology conditions were collected on the Grand River, ON, CA.

base. Underreporting of enteric diseases is considerable because asymptomatic, transient or mildly affected patients may not attend a health care provider (Murphy et al. 2016). Therefore, every unique submitted case to iPHIS was used to maximize the study population. This study population is thought to be representative of the patients suffering impairment from disease. Cases who reported travel outside of the province of Ontario during the disease incubation period, or who could not confirm their travel history during the incubation period, were excluded. All cases in the dataset for the time series were identified as sporadic based on the provincial outbreak numbering system. Cases not identified by the local public health unit as being linked to an outbreak were considered to have occurred independently of other cases and coded with a unique identifier.

\section{Climate and Hydrology Data}

The WHR has a temperate climate consisting of distinct seasons made up of warm summers and cold winters with below freezing temperatures (Peel et al. 2007). Weather and hydrology observations for this region were measured daily over the study period from 1 June 2006 to 31 December 2013. The environmental variables included mean air temperature $\left({ }^{\circ} \mathrm{C}\right)$ and total precipitation $(\mathrm{mm})$, river water level $(\mathrm{m})$ and river water flow rate $\left(\mathrm{m}^{3} / \mathrm{s}\right)$ (Environment and Climate Change Canada 2016a, b). Studies on the physical characteristics of Giardia have found that temperature is an important parameter influencing survival and recovery of viable cysts (Erickson and Ortega 2006). The focus of this study on waterborne transmission meant that precipitation, water flow and water level were variables 
of interest based on our a priori hypothesis. Weather was recorded at the Region of Waterloo International Airport, Waterloo, Ontario; daily water flow and water level observations were collected on the Grand River at Doon, Waterloo County, Ontario (Environment and Climate Change Canada 2016a, b).

\section{Livestock Reservoir Data}

The pilot site of the FoodNet sentinel surveillance program of the Public Health Agency of Canada operated in the WHR between 2005 and 2014. Farm level surveillance of livestock manure samples was conducted. Each month, one or more farm type (dairy, beef or swine farm) was visited and 3 fresh, pooled manure samples and one pooled manure pit sample were collected (Public Health Agency of Canada 2010). Each fresh sample comprised manure from five animals, representing different ages, and the manure pit sample comprised up to five subsamples collected from different depths. Samples were tested by microscopy and PCR. At least one positive sample per farm per collection date underwent further molecular subtyping to determine the assemblage (Public Health Agency of Canada 2010). PCR and/or microscopy results for manure samples were recorded as positive, negative, or not done. A farm was labelled positive if at least one sample from the farm on a given collection date was positive for $G d$ by PCR and/or microscopy. Numeric cyst counts were not performed. Aggregated counts of Giardia-positive farms per manure testing date were compiled to form a time series of positive livestock reservoirs in the region between 1 June 2006 and 31 December 2008.

\section{Data Organization}

The study was separated into two parts to reflect the two objectives: Objective I evaluated associations between human cases and environmental variables between 1 June 2006 and 31 December 2013. For objective II, a subset of observations from Objective I collected between 1 June 2006 and 31 December 2008 were merged with the time series of Giardia-positive farms (livestock reservoir data) to create a dataset that integrated human, animal and environmental data.

\section{Statistical Analysis}

Seasonality

Seasonal fluctuation of human Giardia incidence was assessed for statistical significance by linear combination of sine and cosine terms. We employed methods previously described by $\mathrm{Ng}$ et al. (2008) to use monthly sine and cosine variables to assess seasonality and a year term to evaluate annual trends:

$$
\begin{aligned}
E(Y)= & \exp (\alpha+\beta 1(\text { year })+\beta 2(\sin (2 \pi \text { month } / 12)) \\
& +\beta 3(\cos (2 \pi \text { month } / 12)))
\end{aligned}
$$

where $\alpha$ is a constant, $\beta$ is a regression coefficient for year or month and $E(Y)$ is the expected case count for a given month (Ng et al. 2008).

Annual variation and statistical significance of the seasonal smoothing terms were evaluated using a Poisson regression model with no predictor variables included. Significant smoothing terms $(P \leq 0.05)$ were included in univariable analysis models of predictor variables; oscillating seasonal smoothers (sine and cosine terms) and the year variable were forced into all multivariable models to adjust for the expected confounding effects of seasonal and annual trends.

\section{Multivariable Regression Models}

For each objective, Poisson regression analysis was used to examine temporal associations among average monthly weather conditions (temperature and precipitation), hydrological conditions (river flow and level), and monthly aggregated Giardia-positive farms (Objective II) on monthly aggregated human case counts. Canadian census information provided the total annual population at risk to determine the incidence rate ratio of giardiasis in people in WHR. A 1-month lag period for environmental and livestock reservoir variables was chosen as it included the incubation period of $G d$ in humans (approximately $7-$ 14 days) as well as time for the pathogen to move from livestock and the environment into the human population. Correlation between predictor variables was assessed (cutpoint $>0.8$ ) and highly correlated pairs of variables reduced by eliminating one of the variables from further analysis. After univariable analysis (adjusted for statistically significant seasonal smoothers), monthly averaged environmental variables and monthly aggregated Giardia-positive farms that had incidence rate ratios (IRRs) that met a 
liberal $P$ value $(P \leq 0.2)$ were brought forward into a multivariable Poisson regression model and a backward selection process was used to determine the final multivariable model $(P \leq 0.05)$. Scatter plots of predicted outcomes and Anscombe residuals were created to assess outliers and covariate patterns with high influence on the model.

\section{Case Crossover Models}

A case crossover design was used to assess the impact of acute environmental and livestock exposures on daily human case counts. This approach is useful for rare diseases with a short incubation period and intermittent exposures that create different risk periods through which subjects pass temporally (Maclure 1991; Levy et al. 2001; Ng et al. 2008). The self-matching, case crossover design compares the exposure status immediately before a case occurs to the self-matched exposure status during a control period which is randomly selected to occur prior to, after, or spanning the hazard period (Levy et al. 2001). A time-stratified, 4:1 matched design was used to match four control periods by day of week to the case report date within each 4-week stratum. Conditional logistic regression models were used to assess statistical associations between same week and 14-week-lagged exposures and daily human case counts. Environmental variables were ranked from lowest to highest within each stratum to further elucidate which exposure most contributed to risk of human case development. A distributed lag model was used to account for transmission of the pathogen through the environment and the average incubation period in humans.

Case crossover associations were measured as odds ratios (OR). Statistically significant associations between human cases and exposures reported during the concurrent week (i.e. exposures with no lag period) were not considered to be biologically feasible, given the mean incubation period for $G d$ in people is 7-14 days. All statistical analysis was conducted using STATA 14.0 (STATA Corporation, College Station, TX).

\section{RESULTS}

\section{Objective I: Environmental Variables and Human Case Counts: 2006-2013}

Between 1 June 2006 and December 31st there were 403 cases of giardiasis reported to iPHIS by the WHR. A re- ported symptom onset date was included for 304 (75\%) of the cases. The average annual rate of reported cases was 10.8 cases per 100000 person-years. Monthly human case counts showed a late summer and early autumn peak (Fig. 2). High correlation ( $>0.8$ ) between water level and water flow meant that only water level was examined in multivariable regression analysis.

A statistically significant seasonal pattern (Fig. 2) indicated increased human case occurrence coinciding with the late summer and early autumn period $(P$ value for linear combination of sine and cosine seasonal estimators $=0.01)$. No annual trends were identified $(P>0.05)$.

\section{Poisson Regression Analysis}

Univariable regression analysis found that human disease incidence was inversely associated with water level of the previous month only (IRR $=0.43,95 \%$ CI $0.18,1.00$, $P=0.05$ ) (Table 1). When this environmental exposure was applied to the main effects model adjusted for seasonal predictors and year, it was not statistically significant $(P=0.06)$.

\section{Case Crossover Analysis}

Case crossover analysis found that the water level and flow rate of the Grand River 1 week prior to human cases was associated with an increased risk of giardiasis in the WHR (Table 1). Within-stratum coolest mean air temperatures (lowest tertile) increased the odds of human case occurrence 3 weeks later (OR 0.75, 95\% CI 0.60, 0.94, $P=0.014$ ) (Table 1).

\section{Objective 2: Environmental Variables, Livestock Reservoirs and Human Case Counts, 2006-2008}

Between 1 June 2006 to 31 December 2008, 145 human cases were reported to iPHIS for the WHR representing an average rate of 11.6 cases per 100000 person-years. There were $107(73 \%)$ recorded cases with a reported date of symptom onset. Between 1 June 2006 and 31 December 2008, 335 manure samples were collected from 86 unique cattle and swine farms. 222 (66\%) manure samples tested positive by either microscopy or PCR, or both. Assemblage typing data was restricted to 128 positive samples and identified $30(23 \%)$ samples as assemblages A or B, the remaining samples were typed as assemblage $\mathrm{E}$. 


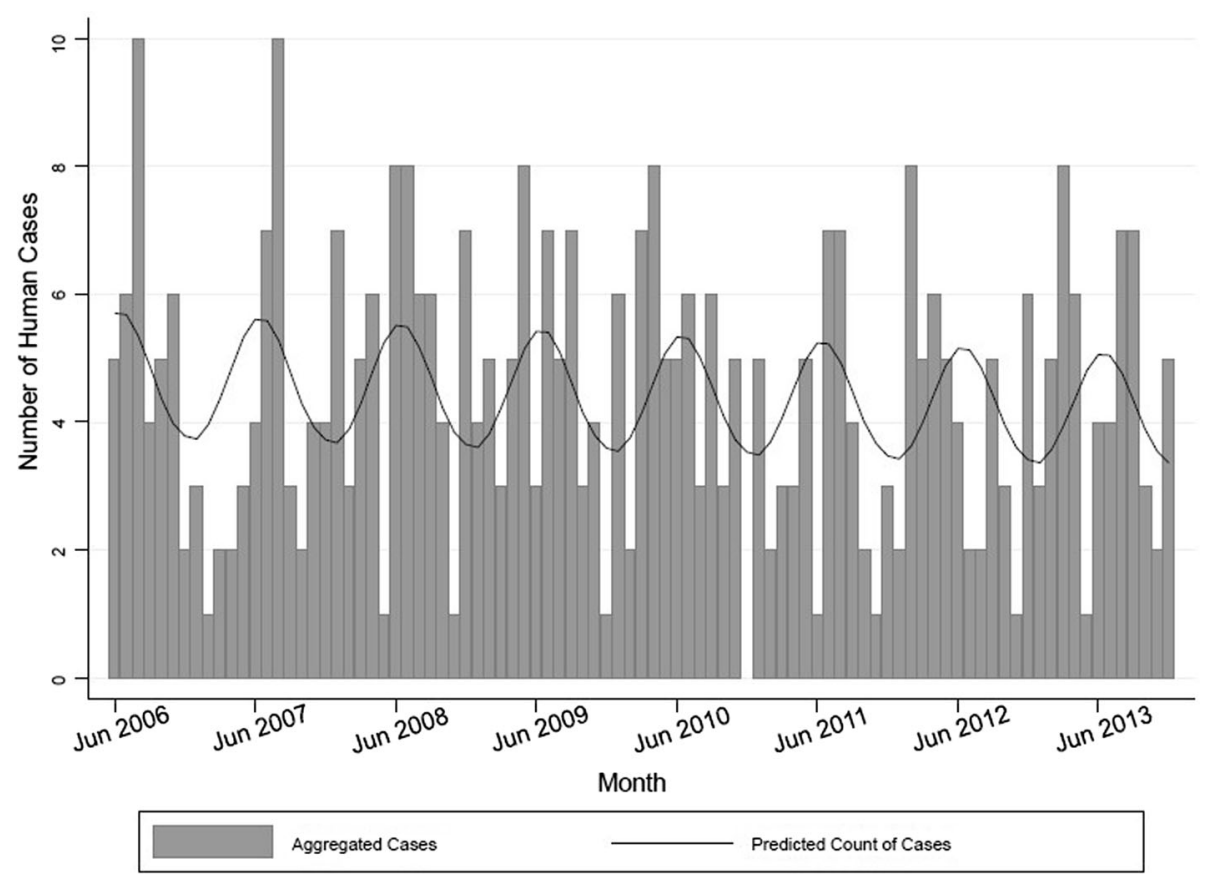

Figure 2. Predicted and observed human Giardia duodenalis cases from the Waterloo health region, Ontario, 2006-2013. Observed monthly human case counts are represented by the grey bars. Model predicted monthly human case counts are represented by the oscillating black line.

Similar to the results of Objective I, the truncated dataset also exhibited a trend of seasonal periodicity for human cases $(P$ value for linear combination of sine and cosine seasonal estimators $=0.002$ ) but no year on year trend $(P>0.05)$.

\section{Poisson Regression Analysis}

For the truncated time series, univariable regression analysis (controlling for seasonality) found that the odds of becoming a case were associated with water level (IRR = 6.18, 95\% CI 1.22, 31.45, $P=0.03)$, 1-month-lagged water level $($ IRR $=0.25,95 \%$ CI $0.04,1.65, P=0.15)$, 1-monthlagged mean temperature $($ IRR $=0.93,95 \%$ CI $0.84,1.02$, $P=0.12$ ), and 1-month-lagged total precipitation (IRR = 1.10, 95\% CI 0.99, 1.21, $P=0.07$ ) (Table 2). A univariable model using the number of Giardia-positive farms as the predictor found that Giardia-positive farms were not associated with human cases $(P>0.05)$. The final multivariable model controlling for seasonality and year, found statistically significant associations with water level and 1month-lagged water level (IRR $=9.80,95 \%$ CI 1.69, 56.71, $P=0.01 ;$ IRR $=0.10,95 \%$ CI $0.01,0.85, P=0.04) \quad($ Table 2).

\section{Case Crossover Analysis}

Case crossover analysis of the truncated time series that included data on livestock reservoirs found associations between mean temperature and increased odds of human cases occurring 3 weeks later (Table 2), whereas decreases in temperature (lowest tertile) for the same lag period reduced the risk of infection (OR 1.58, 95\% CI 1.05, 2.38, $P=0.029$; OR $0.56,95 \%$ CI $0.38,0.83, P=0.004)$. Livestock reservoirs lagged by one week increased human giardiasis risk (OR 1.65, 95\% CI 1.23, 2.22, $P=0.001$ ) (Fig. 3). Increasing levels of precipitation decreased the odds of an individual becoming a case 4 weeks later (OR $0.6,95 \%$ CI $0.37,0.97, P=0.038$ ) (Table 2).

\section{Discussion}

This study contributes to our understanding of the relationship between environmental factors, livestock pathogen reservoirs, and the risk of human giardiasis. Our findings of a late summer peak in incidence are in agreement with previous studies examining temporal trends in human giardiasis (Addiss et al. 1992; Naumova et al. 2007; Keegan et al. 2009; Lal et al. 2012). The consistency of a seasonal pattern, along with the persistence of Giardia as a water- 


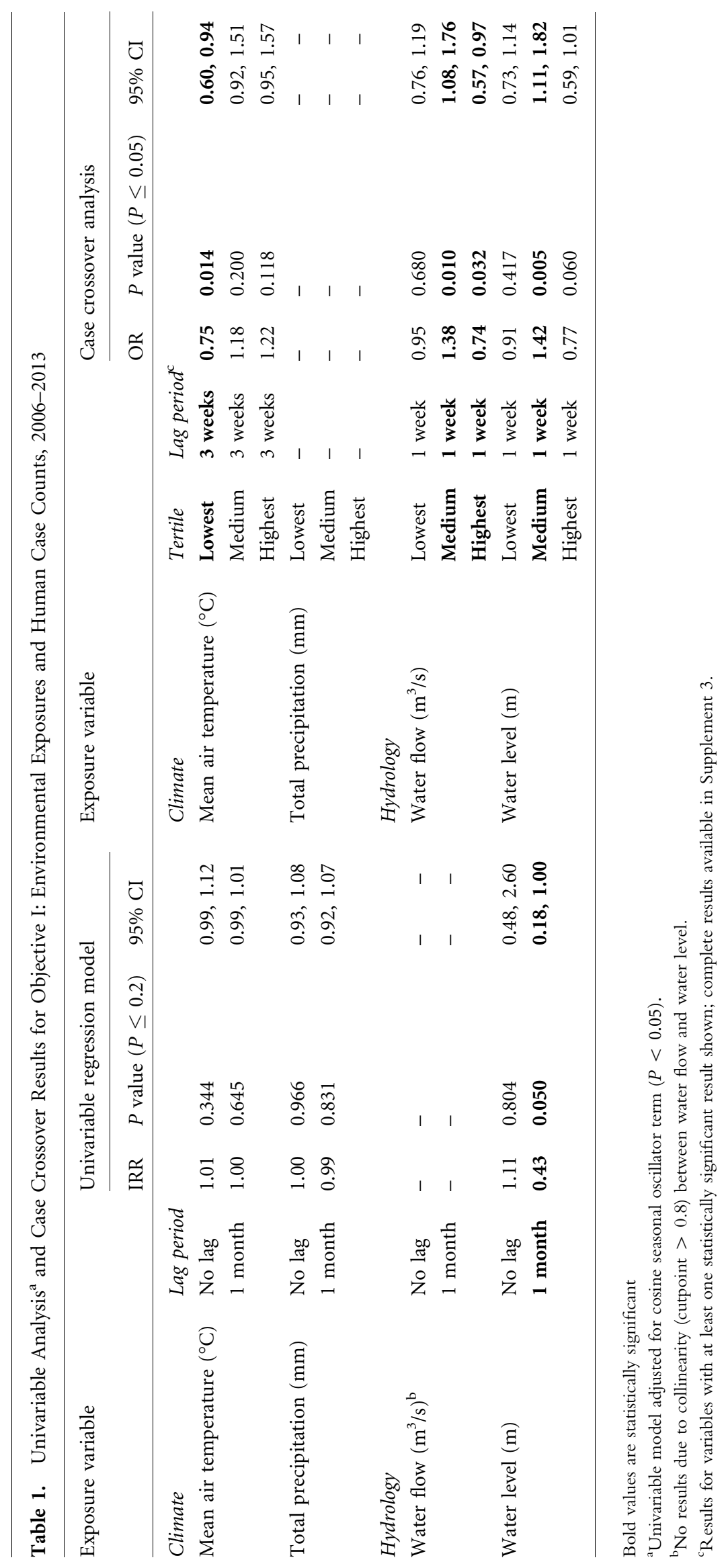




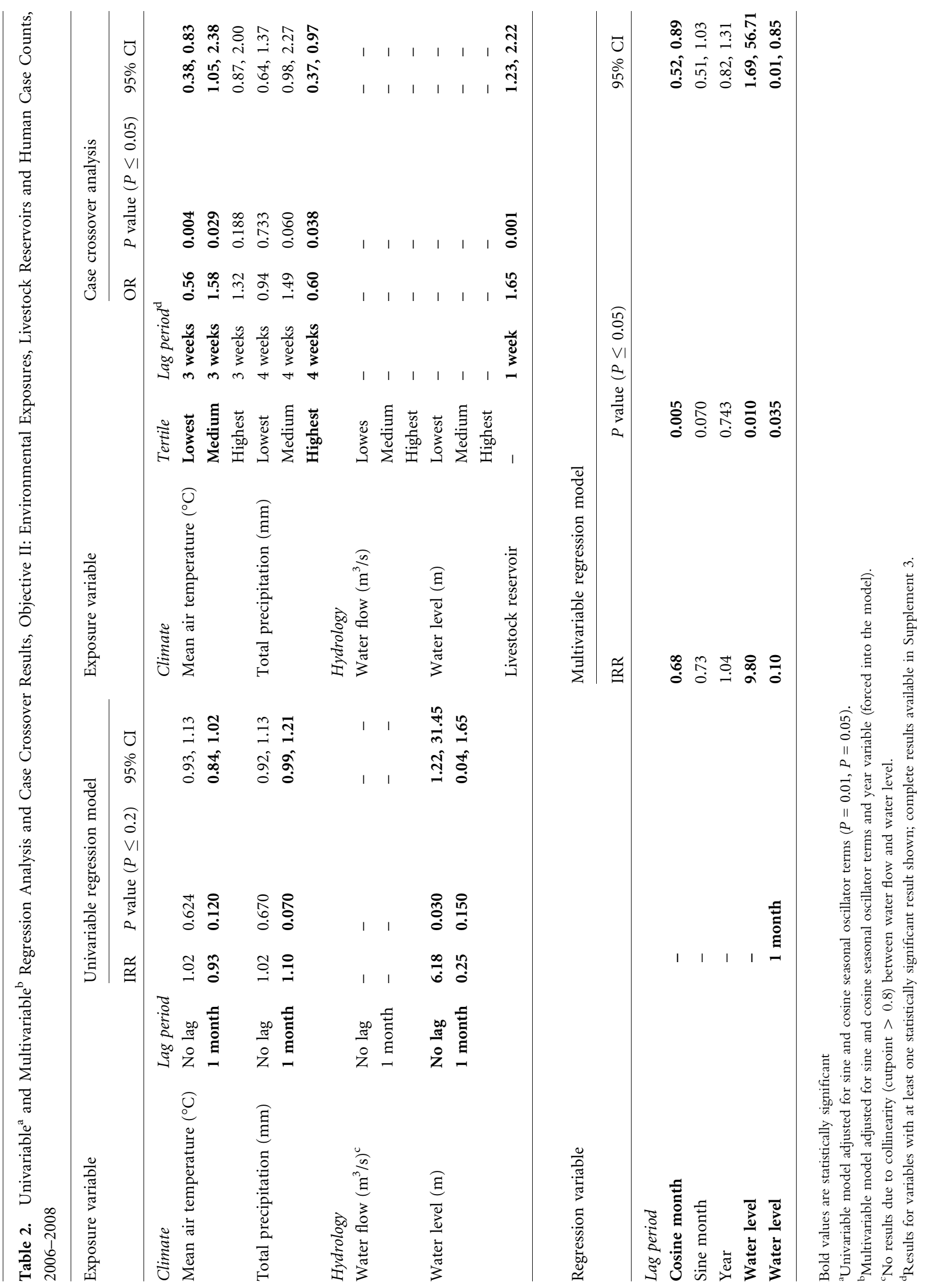




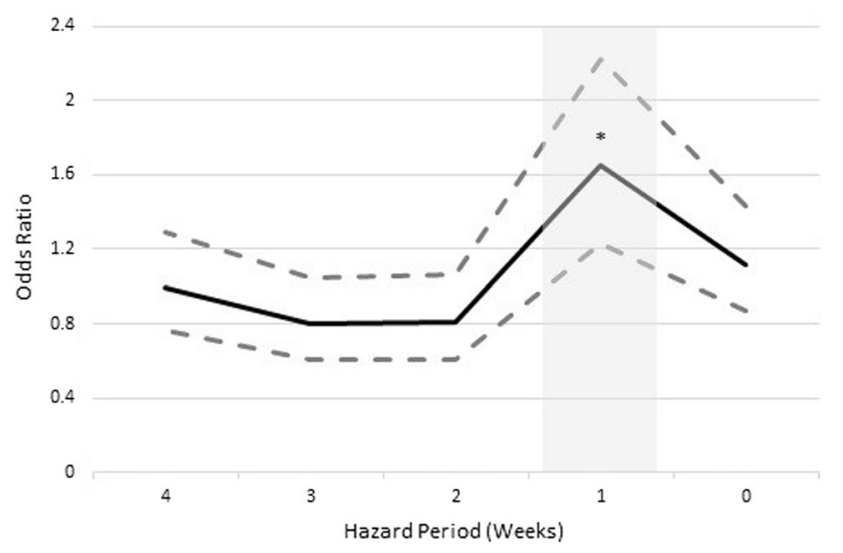

Figure 3. Case crossover results evaluating associations between the presence of weekly aggregated livestock reservoirs of G. duodenalis and human cases of Giardia duodenalis in the Waterloo health region, Ontario, 2006-2008. The hazard period is a 4-week period preceding a human case during which weekly aggregated exposures are assessed for influence on the odds of a human case occurring on day 0 (far right of the $\mathrm{x}$-axis). Solid line $=$ weekly odds ratio, dashed lines = upper and lower $95 \%$ confidence limits, ${ }^{*}=$ statistically significant odds ratio $(P \leq 0.05)$, grey shaded box $=$ time within the hazard period that precedes a human case with a statistically significant exposure.

borne pathogen (Yaoyu and Xiao 2011), suggests human giardiasis is sensitive to climate and hydrology factors.

Associations between human case occurrence and hydrological variables were identified in both the multivariable and case crossover analysis. Inverse associations between aggregated human case counts and river water levels have been previously reported for another waterborne pathogen, Legionella, in multivariable models $(\mathrm{Ng}$ et al. 2008). In contrast to the findings of our multivariable model, the case crossover analysis found that the odds of human cases increased with the water level and flow of the Grand River occurring 1 week before cases. These findings differ from a case crossover study on the enteric protozoa, Cryptosporidium, which identified increased water flow and water levels as having a protective effect on human case risk and attributed this to dilution of pathogen concentrations in the watershed (Brankston et al. 2018). The discrepancy between these results may be related to external control of river flow and level exercised by the Grand River Conservation Authority via a system of dams and water reservoirs which minimize flooding risk and maintain water quality for discharging sewage plants. The Grand River at Doon, Ontario, where hydrology data for this study was collected, is heavily augmented during the late summer dry period.
Up to $90 \%$ of the total river water volume at Doon in August can come from the reservoir (Grand River Conservation Authority 2014). While the effects of river level manipulation may be lost in monthly averaged exposure data, reservoir release seen as an acute exposure could influence associations being found with human case counts using the case crossover methodology. Alternately, resuspension of enteric pathogens from stream sediments during periods of high water flow, such as stormy weather, has been proposed as an explanation for measurements of increased concentrations of E. coli occurring with high stream flow rates (Dorner et al. 2006). While an explanatory hypothesis for a positive association between human giardiasis cases and water levels and flow rates in relation to increased river cyst concentration is unclear in the present study, cyst survival in river sediments and potential for resuspension is an area for future research. When the presence of livestock reservoirs was added to the model (Objective 2), an increase in the odds of human cases was seen with a 1-week lag period. Surveillance studies have confirmed the presence of zoonotic Giardia assemblages in Ontario cattle and swine herds (Coklin et al. 2007; Dixon et al. 2011; Farzan et al. 2011), providing initial support for the hypothesis of spill-over of zoonotic $G d$ from livestock reservoirs. Our results would benefit from molecular typing of Giardia cysts obtained from the Grand River to confirm if potentially zoonotic cysts from livestock can survive effluent transport mechanisms into the watershed.

Statistically significant associations between mean temperature and human case incidence from the case crossover analysis resulted in inverse effects at the lowest tertile of mean temperature with a lag period of 3 weeks. In both the full and truncated time series, the lowest mean air temperatures occurring three weeks prior to human cases decreased the risk of giardiasis.

Previous studies have reported associations between time-lagged high temperatures and increased risk of waterborne enteric disease (Thomas et al. 2006; Naumova et al. 2007; Brankston et al. 2018), possibly reflecting changes in human behaviour and greater opportunities for exposure to contaminated surface waters (Thomas et al. 2006; Peng et al. 2008; Brankston et al. 2018). While the results found in this study do not directly support this hypothesis, it is possible that on days with cooler than average temperatures a protective effect is observed as people may be less inclined to recreate in natural water bodies. 
In the truncated time series, within-stratum highest precipitation levels occurring 4 weeks prior to human cases were inversely related to giardiasis occurrence (Table 2). This result contradicts two studies that found that high rainfall events preceded increased human enteric case incidence (Thomas et al. 2006; Escobedo et al. 2015), however, one study was based in Cuba where average rainfall is higher than what is observed in Ontario (Escobedo et al. 2015). The second study, based in Canada, used a longer hazard period of 6 weeks and stratified precipitation to assess only extreme rainfall events (Thomas et al. 2006). The difference in results may be related to stratification choice, length of hazard period, or pathogen biology.

Some limitations should be noted. Aggregating cases into monthly counts could mask underlying patterns. For example, environmental variables averaged by month may not reflect the true exposures influencing transmission, potentially leading to imprecise inferences. Case crossover analysis aims to avoid this potential bias by examining associations between acute exposures and daily case occurrence using a self-matched study design. However, the testing of multiple hypotheses in the case crossover method may increase the probability of a Type 1 error resulting in spurious associations. In this study, type 1 errors were minimized by grouping exposures to minimize the number of hypotheses tested.

The livestock sampling data for $G d$ was constrained by inconsistent monthly sampling and sporadic genotyping results, leading to assemblage type reporting for just over $50 \%$ of positive samples. We acknowledge that the Giardiapositive farm counts might not accurately reflect the true number of Giardia-positive farms in the region. Nonetheless, the positive counts taken from surveillance data represent a subset and likely underrepresent the true number of cases, influencing the bias towards the null. Assemblage typing data provided confirmation that potentially zoonotic $G d$ assemblages were present in livestock reservoirs in the region, supporting the possibility of a zoonotic transmission route. Molecular studies of isolates and genetic assemblage typing of samples would strengthen the spillover hypothesis. Effect modification between positivetesting farms and environmental variables was not investigated due to limited farm data, however including this as a future direction could contribute to our understanding of livestock disease reservoirs.
Symptom onset dates were reported for $75 \%$ (Objective I dataset) and 73\% (Objective II dataset) of human case counts used in this study. The remaining cases were included in the study to counteract underreporting which is known to occur however, it is possible that the inclusion of these cases resulted in incorrect estimates of associations and exposure lag periods.

Ecological systems are changing at an unprecedented rate (Walther et al. 2002; Post et al. 2009). With climatic conditions expected to change in the coming years, it is necessary to understand the environmental drivers of climate-sensitive diseases (Bouley et al. 2014; Machalaba et al. 2015). While the design of this study precludes conclusions being drawn regarding cause and effect, the relationships described provide an understanding of the associations between human giardiasis, livestock disease reservoirs, and environmental variables using a one health approach.

\section{CONCLUSION}

Seasonality of human $G d$ cases appears to be influenced by hydrology and temperature variables occurring 1-3 weeks prior to case occurrence. Molecular epidemiology confirming survival of zoonotic pathogen spill-over from livestock into watersheds would assist in clarifying the influence of livestock reservoirs. The results of this study contribute information about the influence of environmental and livestock exposures on a climate-sensitive waterborne disease and may be useful in future forecasting of the effects of climate change on human enteric disease.

\section{ACKNOWLEDGEMENTS}

We thank Public Health Ontario, Public Health Agency of Canada and Environment and Climate Change Canada for providing access to the datasets required to undertake this study.

\section{FUNDING}

This study was funded by the Canadian Institutes of Health Research (CIHR) and the Canada Research Chairs Program. 


\section{Compliance with Ethical Standards}

CONFLICT OF INTEREST The authors declare that they have no conflict of interest.

ETHICAL STANDARD The de-identified human case data were retrieved from surveillance databases with no individual identifying information. Human case data are collected according to the Health Protection and Promotion Act, R.S.O. 1990, c. H.7. Individual patient consent is not required to collect such data. This project was approved by the University of Guelph, Research Ethics Board (REB\#15NV011).

\section{Open Access}

This article is distributed under the terms of the Creative Commons Attribution 4.0 International License (http://c reativecommons.org/licenses/by/4.0/), which permits unrestricted use, distribution, and reproduction in any medium, provided you give appropriate credit to the original author(s) and the source, provide a link to the Creative Commons license, and indicate if changes were made.

\section{REFERENCES}

Abdel-Moein KA, Saeed H (2016) The zoonotic potential of Giardia intestinalis assemblage E in rural settings. Parasitology Research 115(8):3197-3202

Addiss DG, Davis JP, et al. (1992) Epidemiology of giardiasis in Wisconsin: Increasing incidence of reported cases and unexplained seasonal trends. Tropical Medicine 47(1):13-19

Bouley T, Gilbert M, et al. (2014) Reducing Climate-Sensitive Disease Risks. Agriculture and Environmental Services Discussion Paper; No. 7. Washington, DC; World Bank Group. http://doc uments.worldbank.org/curated/en/486511468167944431/Reduc ing-climate-sensitive-disease-risks. Accessed June 3, 2017

Brankston G, et al. (2018) Assessing the impact of environmental exposures and Cryptosporidium infection in cattle on human incidence of cryptosporidiosis in Southwestern Ontario. PLoS ONE 13(4):1-12

Cacciò SM, Ryan U (2008) Molecular epidemiology of giardiasis. Molecular and Biochemical Parasitology 160(2):75-80

Coklin T, Farber JM, et al. (2007) Prevalence and molecular characterization of Giardia duodenalis and Cryptosporidium spp. in dairy cattle in Ontario, Canada. Veterinary Parasitology 150(4):297-305

Conlan J, Lal A (2015) Socioeconomic burden of foodborne parasites. In: Foodborne parasites in the food supply web, Gajadhar AA (editor), Cambridge, UK: Elsevier, pp 75-98
Dixon B, Parrington L, et al. (2011) The potential for zoonotic transmission of Giardia duodenalis and Cryptosporidium spp. from beef and dairy cattle in Ontario, Canada. Veterinary Parasitology 175(1-2):20-26

Environment and Climate Change Canada (2016a) Historical climate data archived (2006-2013). http://climate.weather.gc.c a/historical_data/search_historic_data_e.html. Extracted: January 15, 2016

Environment and Climate Change Canada (2016b) Water survey of Canada archived hydrometric data (2006-2013). https://ec.g c.ca/rhc-wsc/default.asp?n=9018B5EC-1. Extracted: January 15, 2016

Erickson MC, Ortega YR (2006) Inactivation of protozoan parasites in food, water, and environmental systems. Journal of food protection 69(11):2786-2808

Escobedo AA, Almirall P, et al. (2015) Potential impact of macroclimatic variability on the epidemiology of giardiasis in three provinces of Cuba, 2010-2012. Journal of Infection and Public Health 8(1):80-89

Farzan A, Parrington L, et al. (2011) Detection and characterization of Giardia duodenalis and Cryptosporidium spp. on swine farms in Ontario, Canada. Foodborne Pathogens and Disease 8(11):1207-1213

Fisman DN (2007) Seasonality of infectious diseases. Annual Review of Public Health 28:127-143

Grand River Conservation Authority (2014) Background Briefings: Reservoir Operations. December 2014:1-2. https://www.grandri ver.ca/en/learn-get-involved/resources/Documents/GRCA_facts heet_Reservoirs.pdf. Accessed May 13, 2017

Greer A, Ng V, et al. (2008) Climate change and infectious diseases in North America: The road ahead. Canadian Medical Association Journal 178(6):715-722

Greig JD, Michel P, et al. (2001) A descriptive analysis of Giardiasis cases reported in Ontario, 1990-1998. Canadian Journal of Public Health 92(5):361-365

Hunter PR, Thompson RCA (2005) The zoonotic transmission of Giardia and Cryptosporidium. International Journal for Parasitology 35(11-12):1181-1190

Keegan VA, Majowicz SE, et al. (2009) Epidemiology of enteric disease in C-EnterNet's pilot site-Waterloo region, Ontario, 1990-2004. Canadian Journal of Infectious Diseases and Medical Microbiology 20(3):79-87

Lal A, Hales S, et al. (2012) Seasonality in human zoonotic enteric diseases: A systematic review. PLoS ONE 7(4):1-11

Levy D, Lumley T, et al. (2001) Referent selection in case-crossover analyses of acute health effects of air pollution. Epidemiology 12(2):186-192

Machalaba C, Romanelli C, et al. (2015) Climate change and health: Transcending silos to find solutions. Annals of Global Health 81(3):445-458

Maclure M (1991) The case-crossover design: A method for studying transient effects on the risk of acute events. American Journal of Epidemiology 133(2):144-153

Murphy HM, Thomas MK, et al. (2016) Estimating the burden of acute gastrointestinal illness due to Giardia, Cryptosporidium, Campylobacter, E. coli $\mathrm{O} 157$ and norovirus associated with private wells and small water systems in Canada. Epidemiology and Infection 144(7):1355-1370

Murray CJL, Vos T, Lozano R, Naghavi M, et al. (2012) Disabilityadjusted life years (DALYs) for 291 diseases and injuries in 21 
regions, 1990-2010: A systematic analysis for the Global Burden of Disease Study 2010. Lancet 380(9859):2197-2223

Naumova EN, Jagai JS, et al. (2007) Seasonality in six enterically transmitted diseases and ambient temperature. Epidemiology and Infection 135(2):281-292

$\mathrm{Ng} \mathrm{V}$, Tank P, et al. (2008) Going with the flow: Legionellosis risk in Toronto, Canada is strongly associated with local watershed hydrology. EcoHealth 5(4):482-490

Ontario Ministry of Health and Long-Term Care (2016) Integrated Public Health Information System, Infectious diseases protocol. http://www.health.gov.on.ca/en/pro/programs/publi chealth/oph_standards/docs/giardiasis_chapter.pdf. Extracted: January 15, 2016

Peel MC, Finlayson BL, et al. (2007) Updated world map of the Köppen-Geiger climate classification. Hydrology and Earth System Sciences 11:1633-1644

Peng X, Murphy T, et al. (2008) Evaluation of the effect of temperature on the die-off rate for Cryptosporidium parvum oocysts in water, soils, and feces. Applied and Environmental Microbiology 74(23):7101-7107

Post E, Forchhammer MC, et al. (2009) Ecological dynamics across the Arctic associated with recent climate change. Science 325(5946):1355-1358

Public Health Agency of Canada (2010) Sample Collection, Preparation and Laboratory Methodologies. National Integrated Enteric Disease Surveillance Program January 2010:1-34. http s://www.canada.ca/en/public-health/services/surveillance/foodn et-canada/sample-collection-preparation-laboratory-methodolo gies.html. Accessed May 24, 2017

Public Health Ontario (2017) Monthly Infectious Diseases Surveillance Report 2017. https://www.publichealthontario.ca/e n/DataAndAnalytics/Documents/PHO_Monthly_Infectious_Di seases_Surveillance_Report_-_February_2017.pdf. Accessed June 23, 2017

Silva RR, da Silva CAM, de Jesus Pereira CA, de Carvalho Nicolato RL, et al. (2009) Association between nutritional status, environmental and socio-economic factors and Giardia lamblia infections among children aged 6-71 months in Brazil. Transactions of the Royal Society of Tropical Medicine and Hygiene. 103(5):512-519

Statistics Canada (2011) Census in agriculture. CANSIM (database). http://www5.statcan.gc.ca/cansim/a03?lang=eng\&patter $\mathrm{n}=004-0200 . .004-0242 \& \mathrm{p} 2=31$. Accessed July 13, 2017

Thomas KM, Charron DF, et al. (2006) A role of high impact weather events in waterborne disease outbreaks in Canada, 1975-2001. International Journal of Environmental Health Research 16(3):167-180

Walther GR, Post E, et al. (2002) Ecological responses to recent climate change. Nature 416(6879):389-395

Wilkes G, Edge TA, et al. (2011) Associations among pathogenic bacteria, parasites, and environmental and land use factors in multiple mixed-use watersheds. Water Research 45:5807-5825

Yaoyu F, Xiao L (2011) Zoonotic potential and molecular epidemiology of Giardia species and giardiasis. Clinical Microbiology Reviews 24(1):110-140 